\section{Filtration, glomeruläre}

\author{
W. G. Guder \\ München, Deutschland
}

Synonym(e) Glomeruläre Clearance; Glomeruläre Filtrationsrate; GFR

\section{Englischer Begriff glomerular filtration rate}

Definition Die glomeruläre Filtration ( $>$ Clearance, glomeruläre) beschreibt den Prozess der Entstehung des Primärfiltrats in der Niere durch die Filtration aus dem kapillären Plasma in den proximalen Tubulus der Niere. Die Gesamtmenge der pro Minute filtrierten Flüssigkeitsmenge wird als glomeruläre Filtrationsrate bezeichnet.

Beschreibung Die glomeruläre Filtration findet in der Basalmembran des Glomerulums statt, einer extrazellulären Matrix, die eine Filtration nach Molekülgröße und Ladung erlaubt. Sie wird durch physikalische Eigenschaften wie Kapillardruck in glomerulären Kapillaren sowie physikalisch-chemischen Eigenschaften der filtrierten Moleküle wie der Oberfläche der Membran und der benachbarten Podozyten beeinflusst. Durch Heparansulfatreste an der den Kapillaren zugewandten Seite kommt es zu negativen Ladungen der Oberfläche, die negativ geladene Proteine von der Filtration abhalten. Dieser Mechanismus ist für Moleküle einer molekularen Größe $>10 \mathrm{kDa}$ bis $<45 \mathrm{kDa}$ relevant (z. B. $>$ Albumin).

Die glomeruläre Clearance wird durch Messung frei filtrierbarer Moleküle im Plasma und im Sammelurin bestimmt (endogen durch Kreatinin [ $>$ Kreatinin-Clearance] und $\triangleright \mathrm{Cy}-$ statin $\mathrm{C}$, exogen durch Inulin [ $>$ Inulin-Clearance] und Iohexol). Neben der Bestimmung der Menge wird zunehmend die Qualität der glomerulären Clearance Gegenstand der diagnostischen Analytik. So wird eine selektive glomeruläre Proteinurie mit Vermehrung ausschließlich von Proteinen $<50 \mathrm{kDa}$ (z. B. Albumin) als Ausdruck einer Störung der Ladungsselektivität der glomerulären Filtration gesehen mit meist guter Prognose, d. h. ohne Verminderung der Filtrationsmenge (z. B. Minimal-change-Nephropathie, IgA-Nephropathie). Eine unselektive glomeruläre Proteinurie mit Ausscheidung von Molekülen $>120 \mathrm{kDa}$ (z. B. IgG, $>$ Transferrin, bis $\mathrm{zu}$ $\checkmark \alpha_{2}$-Makroglobulin) hat demgegenüber eine schlechte Prognose bezüglich der Nierenfunktion (s. a. > Proteinuriediagnostik).

\section{Literatur}

Anders Grubb (2010) Non-invasive estimation of glomerular filtration rate (GFR). The Lund model: Simultaneous use of cystatin C- and creatinine-based GFR-prediction equations, clinical data and an internal quality check. Scandinavian Journal of Clinical and Laboratory Investigation 70(2):65-70

Guder WG, Hofmann W (1991) Glomeruläre Filtration. In: Thomas L, Fateh-Moghadam A, Guder WG et al (Hrsg) Proteindiagnostik. Behring Diagnostika, S 112-123

Hofmann W, Ehrich JHH, Guder WG, Keller F, Scherberich J (2014) Niere und ableitende Harnwege. In: Hofmann W, Aufenanger J, Hoffmann G. Klinikhandbuch Labordiagnostische Pfade, 2. Aufl. Walter de Gruyter, Berlin/Boston, S 130-150

Schleicher E, Nerlich A, Gerbitz KDA (1989) Struktur, Funktion und Pathobiochemie der glomerulären Basalmembran. In: Guder WG, Lang H (Hrsg) Pathobiochemie und Funktionsdiagnostik der Niere. Springer, Berlin/Heidelberg/New York, S 25-44 